\title{
Trophic Interactions of Infant Bifidobacteria and Eubacterium hallii during L-Fucose and Fucosyllactose Degradation
}

\author{
Clarissa Schwab ${ }^{1 *}$, Hans-Joachim Ruscheweyh ${ }^{2,3,4}$, Vera Bunesova ${ }^{1,5}$, Van Thanh Pham ${ }^{1}$, \\ Niko Beerenwinkel ${ }^{2,4}$ and Christophe Lacroix ${ }^{1}$ \\ ${ }^{1}$ Laboratory of Food Biotechnology, Department of Health Sciences and Technology, ETH Zurich, Zurich, Switzerland, \\ ${ }^{2}$ Department of Biosystems Science and Engineering, ETH Zurich, Basel, Switzerland, ${ }^{3}$ Scientific IT Services, ETH Zurich, \\ Basel, Switzerland, ${ }^{4}$ Swiss Institute of Bioinformatics, Basel, Switzerland, ${ }^{5}$ Department of Microbiology, Nutrition, and \\ Dietetics, Czech University of Life Sciences Prague, Prague, Czechia
}

OPEN ACCESS

Edited by:

Andrea Gomez-Zavaglia Center for Research and Development in Food Cryotechnology (CIDCA, CONICET), Argentina

Reviewed by: Atte Von Wright, University of Eastern Finland, Finland Catherine Thornton,

Swansea University, UK

${ }^{*}$ Correspondence:

Clarissa Schwab clarissa.schwab@hest.ethz.ch

Specialty section: This article was submitted to Food Microbiology,

a section of the journal

Frontiers in Microbiology

Received: 02 December 2016 Accepted: 13 January 2017 Published: 30 January 2017

Citation: Schwab C, Ruscheweyh H-J,

Bunesova V, Pham VT, Beerenwinkel N and Lacroix C (2017)

Trophic Interactions of Infant Bifidobacteria and Eubacterium halli during L-Fucose and Fucosyllactose Degradation. Front. Microbiol. 8:95. doi: 10.3389/fmicb.2017.00095
Fucosyllactoses (2'- or $3^{\prime}-\mathrm{FL}$ ) account for up to $20 \%$ of human milk oligosaccharides (HMOs). Infant bifidobacteria, such as Bifidobacterium longum subsp. infantis, utilize the lactose moiety to form lactate and acetate, and metabolize L-fucose to 1,2-propanediol $(1,2-P D)$. Eubacterium hallii is a common member of the adult gut microbiota that can produce butyrate from lactate and acetate, and convert 1,2-PD to propionate. Recently, a Swiss cohort study identified $E$. hallii as one of the first butyrate producers in the infant gut. However, the global prevalence of $E$. hallii and its role in utilization of $\mathrm{HMO}$ degradation intermediates remains unexplored. Fecal 16S rRNA gene libraries $(n=857)$ of humans of all age groups from Venezuela, Malawi, Switzerland, and the USA were screened for the occurrence of $E$. hallii. Single and co-culture experiments of $B$. longum subsp. infantis and $E$. hallii were conducted in modified YCFA containing acetate and glucose, L-fucose, or FL. Bifidobacterium spp. $(n=56)$ of different origin were screened for the ability to metabolize L-fucose. Relative abundance of $E$. hallii was low $\left(10^{-5}-10^{-3} \%\right)$ during the first months but increased and reached adult levels $(0.01-10 \%)$ at $5-10$ years of age in all four populations. In single culture, B. longum subsp. infantis grew in the presence of all three carbohydrates while E. hallii was metabolically active only with glucose. In co-culture $E$. hallii also grew with L-fucose or FL. In co-cultures grown with glucose, acetate, and glucose were consumed and nearly equimolar proportions of formate and butyrate were formed. B. longum subsp. infantis used L-fucose and produced 1,2-PD, acetate and formate in a ratio of 1:1:1, while 1,2-PD was used by $E$. hallii to form propionate. E. hallii consumed acetate, lactate and 1,2-PD released by $B$. longum subsp. infantis from $\mathrm{FL}$, and produced butyrate, propionate, and formate. Beside B. longum subsp. infantis, Bifidobacterium breve, and a strain of $B$. longum subsp. suis were able to utilize L-fucose. This study identified a trophic interaction of infant bifidobacteria and $E$. hallii during L-fucose degradation, and pointed at $E$. hallii as a metabolically versatile species that occurs in infants and utilizes intermediates of bifidobacterial $\mathrm{HMO}$ fermentation.

Keywords: Eubacterium hallii, trophic interactions, bifidobacterium, fucose, fucosyllactose 


\section{INTRODUCTION}

Human milk oligosaccharides (HMOs) are one of the major glycan source of the infant gut microbiota. Primary components of HMOs are D-glucose, D-galactose, L-fucose, $\mathrm{N}$-acetylglucosamine, and sialic acid. Lactose constitutes the reducing end of HMOs, its galactose moiety can be fucosylated or sialylated to form $2^{\prime}$ - or $3^{\prime}$-fucosyllactose $\left(2^{\prime}\right.$-FL or $3^{\prime}$-FL), or $3^{\prime}$ - and $6^{\prime}$-sialyl-lactose ( $3^{\prime}$-SL or $6^{\prime}$-SL). Lactose can also be elongated with units of $\mathrm{N}$-acetyllactosamine (Gal- $\beta 1-4 \mathrm{GlcNAc})$ with its simplest form being Lacto-N-neotetraose (LNnT) (Kunz et al., 2000). The composition of HMOs is individually different and remains stable during different lactation phases (Niñonuevo et al., 2008; de Leoz et al., 2012). FLs can account for up to $20 \%$ of all HMOs (Niñonuevo et al., 2008; de Leoz et al., 2012).

Infant bifidobacteria species, such as Bifidobacterium longum subsp. infantis and Bifidobacterium bifidum, are adapted to degrade HMOs (LoCascio et al., 2010; Rockova et al., 2012; Turroni et al., 2014) and constitute a big proportion of the infant intestinal microbiota immediately after birth (Avershina et al., 2013; Jost et al., 2014). The degradation of HMOs relies on a complex network of extracellular solute binding proteins, transporters, and intra- or extracellular glycosyl hydrolases (GH). $B$. longum subsp. infantis carries out intracellular degradation while B. bifidum metabolizes HMOs extracellularly (Garrido et al., 2015). Both B. longum subsp. infantis and B. bifidum possess several $\alpha$-fucosidases that release L-fucose from FL (Sela et al., 2012; Garrido et al., 2015). L-fucose can then be either used by the strain itself or can be released for bacterial cross-feeding (Garrido et al., 2015; Bunesova et al., 2016).

L-fucose is a desoxyhexose that is a common component of many $\mathrm{N}$ - and O-linked glycans and of glycolipids produced by mammalian cells (Becker and Lowe, 2003). L-fucose utilization has been investigated in depth in pathogens such as Escherichia coli, Shigella spp. and Bacillus cereus (Staib and Fuchs, 2014). These bacteria employ a fucose isomerase FucI, a fucose aldolase FucA, and a fucose kinase FucK to form L-lactaldehyde which can be further metabolized to 1,2-propanediol (1,2-PD). Recently, we could show that strains of $B$. longum subsp. infantis and $B$. longum subsp. suis metabolize L-fucose to 1,2-PD presumably by a pathway that employs non-phosphorylated intermediates similar to Campylobacter and Xanthomonas spp. (Bunesova et al., 2016). Genes related to L-fucose degradation were located on two genomic regions, one is part of the HMO degradation cluster H1 of B. longum subsp. infantis DSM 20088 (LoCascio et al., 2010), while region 2 contained a gene encoding a putative fucose permease (Bunesova et al., 2016).

In the intestine, 1,2-PD is a precursor of propionate by a pathway that employs a glycerol/diol dehydratase as a key enzyme (Reichardt et al., 2014). The frequent detection of genes encoding glycerol/diol dehydratases in fecal metagenomes of adults suggested that 1,2-PD conversion significantly contributes to intestinal propionate formation (Reichardt et al., 2014; Engels et al., 2016). One species with the ability to convert 1,2-PD to propionate is Eubacterium hallii which is a common commensal in adults (Engels et al., 2016). E. hallii formed similar amounts of propionate in the presence or absence of glucose and did not utilize glucose if 1,2-PD was present (Engels et al., 2016). E. hallii can also grow and form butyrate using either glucose, or acetate and lactate as substrates (Duncan et al., 2004).

We hypothesized that a trophic interaction between $E$. hallii and $B$. longum subsp. infantis can yield short chain fatty acids (SCFAs) butyrate or propionate from lactate and acetate, or from 1,2-PD, respectively. Both butyrate and propionate are important for gut microbiota/host homeostasis as they interact with the host epithelium and impact the immune system. Butyrate is a main energy source of colonocytes, impacts cell proliferation and differentiation, and lowers the risk of colitis and colorectal cancer (Wong et al., 2006; Plöger et al., 2012). Propionate acts as a precursor for gluconeogenesis in the liver and also impacts cell differentiation with potential health-promoting impact on intestinal inflammation, and cancer development (Reichardt et al., 2014).

Bifidobacterium is the predominant genus of the gut microbiota of breast fed infants. Little data exists on the occurrence of E. hallii in early life. Recently, a Swiss cohort study identified $E$. hallii as one of the first butyrate producers in the infant gut (Pham et al., 2016). However, the global prevalence of E. hallii and its role in the metabolism of L-fucose sourced from HMOs remains unexplored.

It was therefore the aim of this study to investigate the occurrence and abundance of $E$. hallii in populations of different age and origin, and to prove trophic interactions of $E$. hallii and $B$. longum subsp. infantis during growth in the presence of glucose, L-fucose, and FL. We also screened further Bifidobacterium spp. and strains $(n=56)$ to investigate whether species other B. longum subsp. infantis or subsp. suis are able to metabolize L-fucose.

\section{METHODS}

\section{Bacterial Strains and Culture Conditions}

E. hallii DSM 3353 obtained from the Deutsche Sammlung von Mikroorganismen und Zellkulturen (DSMZ) was cultivated in modified YCFA medium (mYCFA) containing $30 \mathrm{mM}$ acetate as described by Duncan et al. (2004) with slight modifications (Table 1). All components except L-cysteine-HCL (Sigma-Aldrich) were solubilized in deionized water, and $\mathrm{pH}$ was adjusted to $\mathrm{pH} 7.6$ with $\mathrm{NaOH}$. The medium was flushed with $\mathrm{CO}_{2}$ and boiled. When the color changed from blue to pink, L-cysteine- $\mathrm{HCl}(0.01 \%$, w/v) was added. The medium was transferred to Hungate tubes flushed with $\mathrm{CO}_{2}$, and tubes were sealed and autoclaved. Stab cultures of $E$. hallii that were frozen at $-20^{\circ} \mathrm{C}$ in mYCFA agar (1.5\% (w/v) agar) were used as stock cultures. For each experiment, a fresh agar stock was thawed; $1 \mathrm{ml}$ of liquid YCFA medium was added and thoroughly shaken before being transferred to $8 \mathrm{ml}$ liquid mYCFA medium. After incubation at $37^{\circ} \mathrm{C}$ for $24 \mathrm{~h}$, the culture was transferred at least once to fresh mYCFA broth before the experiment. Bifidobacterium spp. (Table 2) were obtained from the culture collections of the Laboratory of Food Biotechnology of ETH Zurich, the Department of Microbiology, Nutrition, and Dietetics, of the Czech University of Life Sciences Prague, or 
TABLE 1 | mYCFA medium composition.

\begin{tabular}{|c|c|}
\hline Component & Addition \\
\hline Amicase & $1 \%(w / v)$ \\
\hline Yeast extract & $0.25 \%(w / v)$ \\
\hline Sodium bicarbonate & $0.5 \%(\mathrm{w} / \mathrm{v})$ \\
\hline Glucose (replaced with L-fucose and FL) & $1 \%(w / v)$ \\
\hline $\begin{array}{l}\text { Mineral solution [3\% (w/v) potassium dihydrogen phosphate, } 6 \% \\
(\mathrm{w} / \mathrm{v}) \text { sodium chloride, } 0.6 \%(\mathrm{w} / \mathrm{v}) \text { magnesium sulfate, } 0.6 \% \\
\text { calcium chloride }(\mathrm{w} / \mathrm{v})]\end{array}$ & $15 \%(v / v)$ \\
\hline $\begin{array}{l}\text { Vitamin solution }[0.01 \%(\mathrm{w} / \mathrm{v}) \text { biotin, } 0.01(\mathrm{w} / \mathrm{v}) \text { cobalamin, } 0.03 \% \\
\mathrm{p} \text {-aminobenzoic acid }(\mathrm{w} / \mathrm{v}), 0.05 \% \text { folic acid }(\mathrm{w} / \mathrm{v}), 0.15 \% \\
\text { pyridoxamine }(\mathrm{w} / \mathrm{v})]\end{array}$ & $0.1 \%(v / v)$ \\
\hline $\begin{array}{l}\text { Volatile fatty acid mix [56.6\% (v/v) acetic acid, } 20 \%(\mathrm{v} / \mathrm{v}) \text { butyric } \\
\text { acid, } 13.3 \%(\mathrm{v} / \mathrm{v}) \text { propionic acid] }\end{array}$ & $0.31 \%(v / v)$ \\
\hline Hemin $\left(0.5 \mathrm{mg} \mathrm{ml}^{-1}\right)$ & $0.02 \%$ \\
\hline Resazurin (1 $\mathrm{mg} \mathrm{ml}^{-1}$ ) & $0.1 \%$ \\
\hline L-cysteine hydrochloride monohydrate & $0.1 \%$ \\
\hline
\end{tabular}

from DSMZ. Stock cultures of bifidobacteria were maintained at $-80^{\circ} \mathrm{C}$ in $30 \%$ glycerol. To prepare working cultures, bifidobacteria were streaked on Wilkens-Chalgren medium (Oxoid) supplied with soya peptone $\left(5 \mathrm{~g} \mathrm{~L}^{-1}\right.$, Biolife, WCSP), Tween 80 ( $1 \mathrm{~mL} \mathrm{~L}^{-1}$, Sigma-Aldrich), and fresh, filter-sterilized L-cysteine-HCl $\left(0.5 \mathrm{~g} \mathrm{~L}^{-1}\right)$. Single colonies were picked and were grown in liquid WCSP supplied with fresh, filter-sterilized L-cysteine- $\mathrm{HCl}\left(0.5 \mathrm{~g} \mathrm{~L}^{-1}\right)$ at $37^{\circ} \mathrm{C}$ for $24 \mathrm{~h}$. For preparation of co-culture experiments, $100 \mu \mathrm{L}$ of B. longum subsp. infantis overnight culture grown liquid WCSP were added to mYCFA medium, and the culture was incubated at $37^{\circ} \mathrm{C}$ for $24 \mathrm{~h}$. Unless otherwise stated, mYCFA containing $55 \mathrm{mM}$ glucose was used to routinely cultivate $E$. hallii and $B$. longum subsp. infantis.

\section{Single and Co-Culture Studies in the Presence of Different Substrates}

Growth kinetics were assessed in mYCFA medium supplied with glucose (50 mM, mYCFA_glc, Sigma-Aldrich), L-fucose ( $40 \mathrm{mM}$, mYCFA_fuc, Sigma-Aldrich), or FL (6 mM 2'-FL and $6 \mathrm{mM} \mathrm{3'-}$ FL, mYCFA_FL, Glycom A/S). Trace amounts of glucose were added to mYCFA_fuc to enforce initial growth (Bunesova et al., 2016). Hungate tubes containing $9 \mathrm{ml}$ mYCFA_glc, mYCFA_fuc, or mYFA_FL were inoculated with overnight cultures of $E$. hallii and $B$. longum subsp. infantis ( $0.25 \mathrm{~mL}$ each). For comparison, $E$. hallii and $B$. longum subsp. infantis were also grown in single cultures. Samples were taken after $0,4,8,12,24$, and $48 \mathrm{~h}$ of incubation for substrate and metabolite analysis, and for DNA isolation. Bacterial growth was evaluated by measuring the optical density at $600 \mathrm{~nm}\left(\mathrm{OD}_{600}\right)$. Additionally, $16 \mathrm{~S}$ rRNA gene copies of $E$. hallii and B. longum subsp. infantis were determined in the co-cultures as outlined below. Growth was investigated in at least independent triplicates with the exception of B. longum subsp. infantis growth in mYCFA_glc and mYCFA_fuc, which was only investigated in duplicates. Therefore, standard deviations are not shown in the respective graphs.

\section{Screening of Bifidobacteria Strains for L-Fucose Utilization}

Overnight bifidobacteria cultures grown in liquid WCSP were washed and resuspended in phosphate buffered saline (PBS). Bifidobacteria $(50 \mu \mathrm{L})$ were inoculated in $950 \mu \mathrm{L}$ API medium supplied fresh, filter-sterilized L-cysteine- $\mathrm{HCl}\left(0.5 \mathrm{~g} \mathrm{~L}^{-1}\right)$ and with $30 \mathrm{mM}$ glucose, or with $30 \mathrm{mM} \mathrm{L}$-fucose and trace amounts of glucose. Cultures were incubated at $37^{\circ} \mathrm{C}$ for $48 \mathrm{~h}$. Growth and utilization of the carbohydrate source was judged by color change of the medium from blue to yellow. For selected strains, L-fucose utilization and metabolite formation were assessed by HLPC-RI as outlined below.

\section{Analysis of Substrate Utilization and Metabolite Formation}

Glucose and L-fucose consumption, and the formation of 1,2-PD, lactate, acetate, formate, butyrate, and propionate was measured using high performance liquid chromatography (Merck-Hitachi) equipped with an Aminex HPX-87H column $(300 \times 7.8 \mathrm{~mm}$; BioRad) and a refractive index detector (HPLC-RI). Samples were centrifuged at $13,000 \mathrm{~g}$ for $5 \mathrm{~min}$ at $4^{\circ} \mathrm{C}$. Supernatants (40 $\mu \mathrm{L}$ injection volume) were eluted with $10 \mathrm{mM} \mathrm{H}_{2} \mathrm{SO}_{4}$ at a flow rate of $0.6 \mathrm{ml} \mathrm{min}^{-1}$ at $40^{\circ} \mathrm{C}$. Sugars, SCFAs, 1,2-PD, and lactate (all Sigma-Aldrich) were quantified using external standards.

Propanal and propanol were quantified with ion chromatography with pulsed amperometric detection (ICPAD) on a ICS- $5000^{+}$system (Thermo Scientific) equipped with a quaternary gradient pump, a thermoautosampler, and an electrochemical detector with a cell containing an $\mathrm{Ag} / \mathrm{AgCl}$ reference electrode and a disposable thin-film platinum working electrode tempered at $25^{\circ} \mathrm{C}$. Analytes were separated on a IonPac ICE-AS1 $4 \times 250 \mathrm{~mm}$ ion-exclusion column with guard column (Thermo Scientific) operated at $30^{\circ} \mathrm{C}$ using isocratic conditions (0.1 M methanesulfonic acid; $0.2 \mathrm{~mL} \mathrm{~min}^{-1}$ ) for 36 min. The injection volume was $10 \mu \mathrm{L}$. Electrochemical data was obtained using a triple potential waveform consisting of regeneration/detection, oxidation, and reduction potentials: $E_{1}=0.3 \mathrm{~V}\left(t_{1}=0.31 \mathrm{~s}\right), E_{2}=1.25 \mathrm{~V}\left(t_{2}=0.34 \mathrm{~s}, t_{\text {int }}=0.02\right.$ $\mathrm{s}), E_{3}=-0.4 \mathrm{~V}\left(t_{3}=0.39 \mathrm{~s}\right)$. Currents were measured and integrated with respect to time $\left(t_{\text {int }}\right)$. Propanal and propanol (Sigma-Aldrich) were quantified using external standards.

\section{DNA Isolation and Quantification E. hallii and $B$. longum subsp. Infantis in Infant Feces and in Co-Cultivation Studies}

Genomic DNA was isolated from $0.5 \mathrm{~mL}$ fermented mYCFA using the FastDNA SPIN Kit for Soil (MP Biomedicals). Genomic DNA from stool samples $(n=368)$ collected as part of an infant cohort study, and of Swiss children and adult had been isolated as described before (Vanderhaeghen et al., 2015; Pham et al., 2016). The abundance of $E$. hallii was determined using primers EhalF (5' - GCGTAGGTGGCAGTGCAA - $3^{\prime}$ ) and EhalR (5' - GCACCG RAGCCTATACGG-3') (Ramirez-Farias et al., 2009). B. longum subsp. infantis was quantified using primer pair $\mathrm{F}$ ( $5^{\prime}$-TCGCGT CYGGTGTGAAAG-3'), and R (5'-CCACATCCAGCRTCCAC$\left.3^{\prime}\right)$ (Rinttilä et al., 2004). Primers Eub338F (5'-ACTCCTACG 
TABLE 2 | L-fucose utilization and 1,2-PD formation of strains of bifidobacteria.

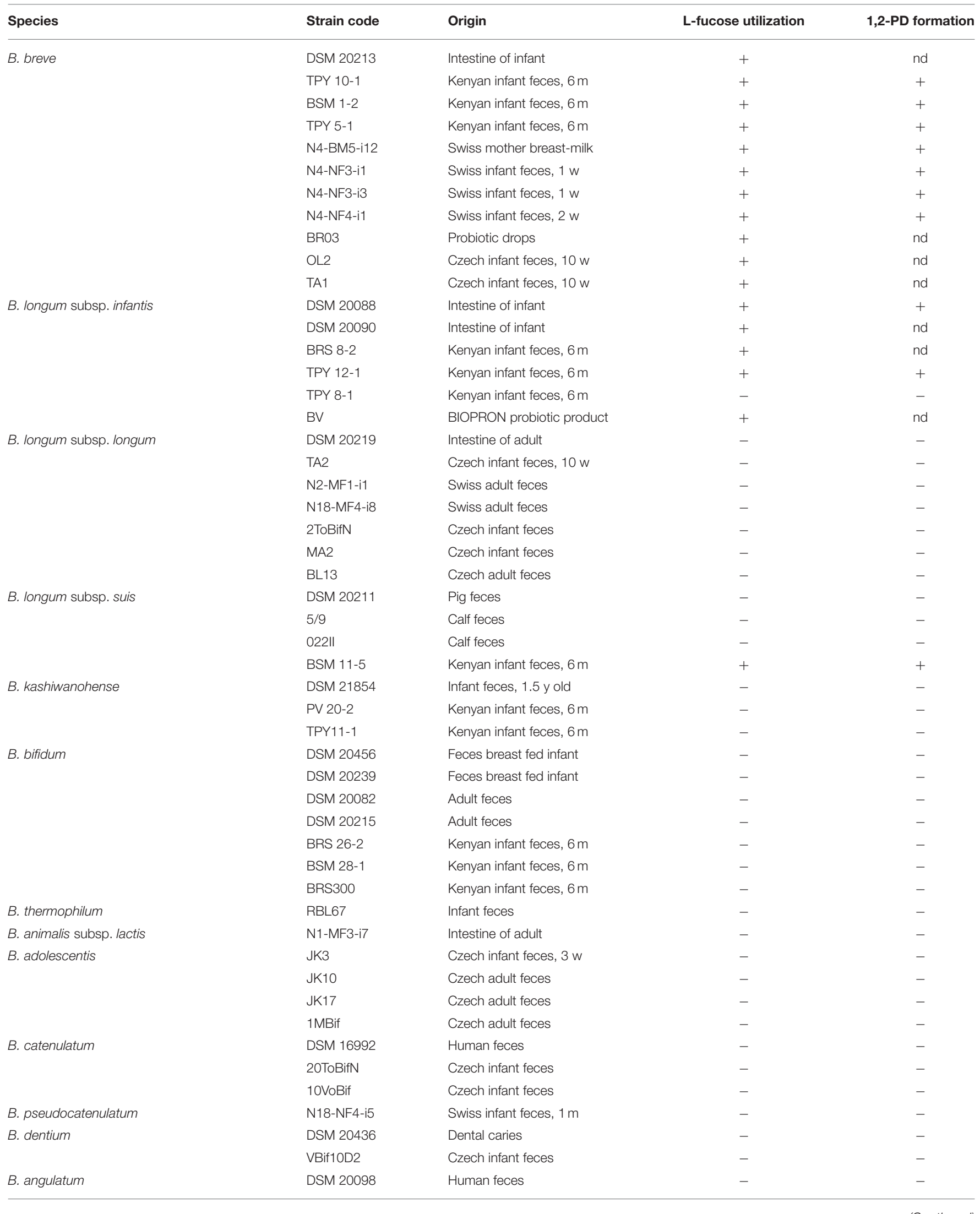


TABLE 2 | Continued

\begin{tabular}{|c|c|c|c|c|}
\hline Species & Strain code & Origin & L-fucose utilization & 1,2-PD formation \\
\hline B. minimum & DSM 20102 & Sewage & - & - \\
\hline \multirow[t]{2}{*}{ B. pseudolongum subsp. pseudolongum } & DSM 20099 & Pig feces & - & - \\
\hline & DSM 20095 & Chicken feces & - & - \\
\hline \multirow[t]{2}{*}{ B. pseudolongum subsp. globosum } & DSM 20092 & Rumen & - & - \\
\hline & PV8-2 & Kenyan infant feces, $6 \mathrm{~m}$ & - & - \\
\hline
\end{tabular}

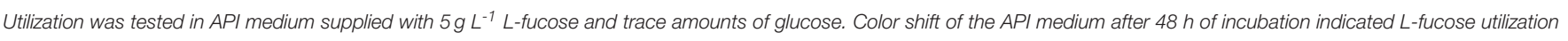
(+). 1,2-PD formation was verified by HPLC-Rl for selected samples (Table 3). nd, not determined; m, months; w, weeks.

GGAGGCAGCAG-3') and Eub518R (5'- ATTACCGCGGCT GCTGG- $3^{\prime}$ ) were employed to quantify total bacteria $16 \mathrm{~S}$ rRNA genes (Fierer et al., 2005). Reactions were performed using a 7500 Fast Real-Time PCR System (Applied Biosystems) and the Kapa SYBR FAST qPCR mastermix (Biolab Scientifics Instruments SA). Thermal cycling started with an initial denaturation step at $95^{\circ} \mathrm{C}$ for $3 \mathrm{~min}$, followed by 40 cycles consisting of denaturation at $95^{\circ} \mathrm{C}$ for $3 \mathrm{~s}$, annealing at $60^{\circ} \mathrm{C}$ for $10 \mathrm{~s}$, and elongation at $72^{\circ} \mathrm{C}$ for $25 \mathrm{~s}$. To verify specificity of amplification, melting curve analysis and agarose gel electrophoresis for amplicon size control were performed. To generate standards, PCR amplicons were cloned into PGEMT Easy Vector and heterologously expressed in E. coli according to instructions of the supplier (Promega). Standard curves were prepared from ten-fold dilutions of linearized plasmids harboring the $16 \mathrm{~S}$ rRNA gene of interest. Linear detection range was between, $\log 2.3$ and $\log 8.3$ gene copies for E. hallii 16S rRNA genes, between log 2.9 and $\log$ 8.9 gene copies bifidobacteria 16S rRNA genes, and between $\log 3.0$ and $\log 8.0$ gene copies for total bacteria 16S rRNA genes. A factor of 5.5 and 4 for Eubacterium spp. and B. longum subsp. infantis, (rrnDB, http://rrndb.mmg.msu.edu; Větrovský and Baldrian, 2013), respectively, was applied to calculate the numbers of cells accounting for several 16S rRNA gene copies per genome.

\section{6s rRNA Gene Amplicon Libraries Screens} 16S rRNA gene sequencing datasets published by Yatsunenko et al. (2012) were downloaded from MG-RAST (MG-RAST ID 401). The 489 datasets with known age of the donor contained in total $1.0^{*} 10^{9}$ sequences and on average $2.1^{*} 10^{6}$ sequences. All reads were aligned against the Silva database (version 123.1, Quast et al., 2013) using MALT in semiglobal alignment mode (Herbig et al., 2016) and only matches with a percent identity $>97 \%$ were reported. Alignments were then used to assign reads on the Silva taxonomy. A read was placed on the lowest taxon so that at least $90 \%$ of the alignments were covered by that taxon (majority vote $90 \%$ ). Unaligned reads were extracted and placed on the Silva taxonomy by using the rdp classifier with a cutoff of 0.8 .

\section{Identification of L-Fucose Utilization Related Genes in B. breve Genomes}

Genomes of Bifidobacterium breve DSM 20213 (PRJDB57) and UCC 2003 (CP000303.1) were screened for genes encoding proteins related to L-fucose metabolism using BlastP and the corresponding proteins of B. longum subsp. infantis DSM 20088 region 1 and 2 for the query (Bunesova et al., 2016, see also below).

\section{Screening of Shotgun Sequencing Datasets for Presence of Proteins Encoding L-Fucose Utilization Regions 1 and 2}

Metagenomic datasets $(n=111)$ published by Yatsunenko et al. (2012) were downloaded from MG-RAST (MG-RAST ID 98). The datasets contained in total 16,318,166 sequences and on average 147,010 sequences per sample. The smallest and largest datasets contained 19,587 and 478,588 sequences, respectively, with an average sequence length of 358 bases. All datasets were aligned using DIAMOND blastx in sensitive mode (Buchfink et al., 2015) against a modified bacterial RefSeq database (Pruitt et al., 2007). The modified RefSeq database was composed by adding 24 protein sequences of $B$. longum subsp. infantis DSM 20088 and $B$. breve DSM 20213 fucose utilization regions 1 and 2 (Bunesova et al., 2016, WP_003830405.1, WP_003830403.1, WP_003830401.1, WP_003830400.1, WP_065457149.1, WP_014484327.1, WP_003829769.1, WP_003829768.1, WP_025300063.1, WP_013141362.1, WP_003829764.1, WP_012578562.1, WP_012578563.1, WP_012578564.1, WP_012578565.1, WP_012578566.1, WP_012578567.1, WP_012578568.1, WP_013141357.1, WP_012578533.1, WP_012578534.1, WP_012578535.1, WP_012578536.1, WP_012578537.1) to the standard RefSeq database. Reads were assigned to the best aligning protein sequences which had a bitscore $>50$. Reads assigned to the 24 proteins sequences of interest were reported.

\section{Statistical Analysis}

A sigmoidal non-linear regression model [Weibull, 5 Parameter model, $\mathrm{f}=\mathrm{if}\left(\mathrm{x}<=\mathrm{x} 0-\mathrm{b}^{*} \ln (2)^{\wedge}(1 / \mathrm{c}) ; \mathrm{y} 0 ; \mathrm{y} 0+\mathrm{a}^{*}(1-\exp (-(\mathrm{abs}(\mathrm{x}-\right.$ $\left.\left.\left.\left.\mathrm{x} 0+\mathrm{b}^{*} \ln (2)^{\wedge}(1 / \mathrm{c})\right) / \mathrm{b}\right)^{\wedge} \mathrm{c}\right)\right)$ ) implemented in SigmaPlot 13, Systat Software] was chosen to fit curves to $\log \%$ abundance data of $E$. hallii in the different populations. Student's paired $t$-test with two-tailed distribution was used to identify significant differences in metabolite formation, $\mathrm{OD}_{600 \mathrm{~nm}}$, and cell counts between treatments. A $p<0.05$ was considered significant. 


\section{RESULTS}

\section{Age and Geographical-Dependent Occurrence of $E$. hallii}

We screened 489 previously obtained 16S rRNA gene amplicon libraries from Malawi, Venezuela and USA (Yatsunenko et al., 2012) for the occurrence of E. hallii. Concurrently, we determined E. hallii relative abundance in a Swiss infant cohort study which followed the fecal microbiota of 40 infants for the first 2 years of life (Pham et al., 2016) using qPCR, and compared to Swiss teenagers and Swiss adults (Vanderhaeghen et al., 2015; together $n=368$; Figure 1). Minimum detection limits were $\sim 10^{-4}$ and $10^{-5} \%$ relative abundance for $16 \mathrm{~S}$ rRNA gene libraries and qPCR screenings, respectively.

In populations from Venezuela, the USA, and Malawi, E. hallii occurred in all infant samples of 1 year of age or younger

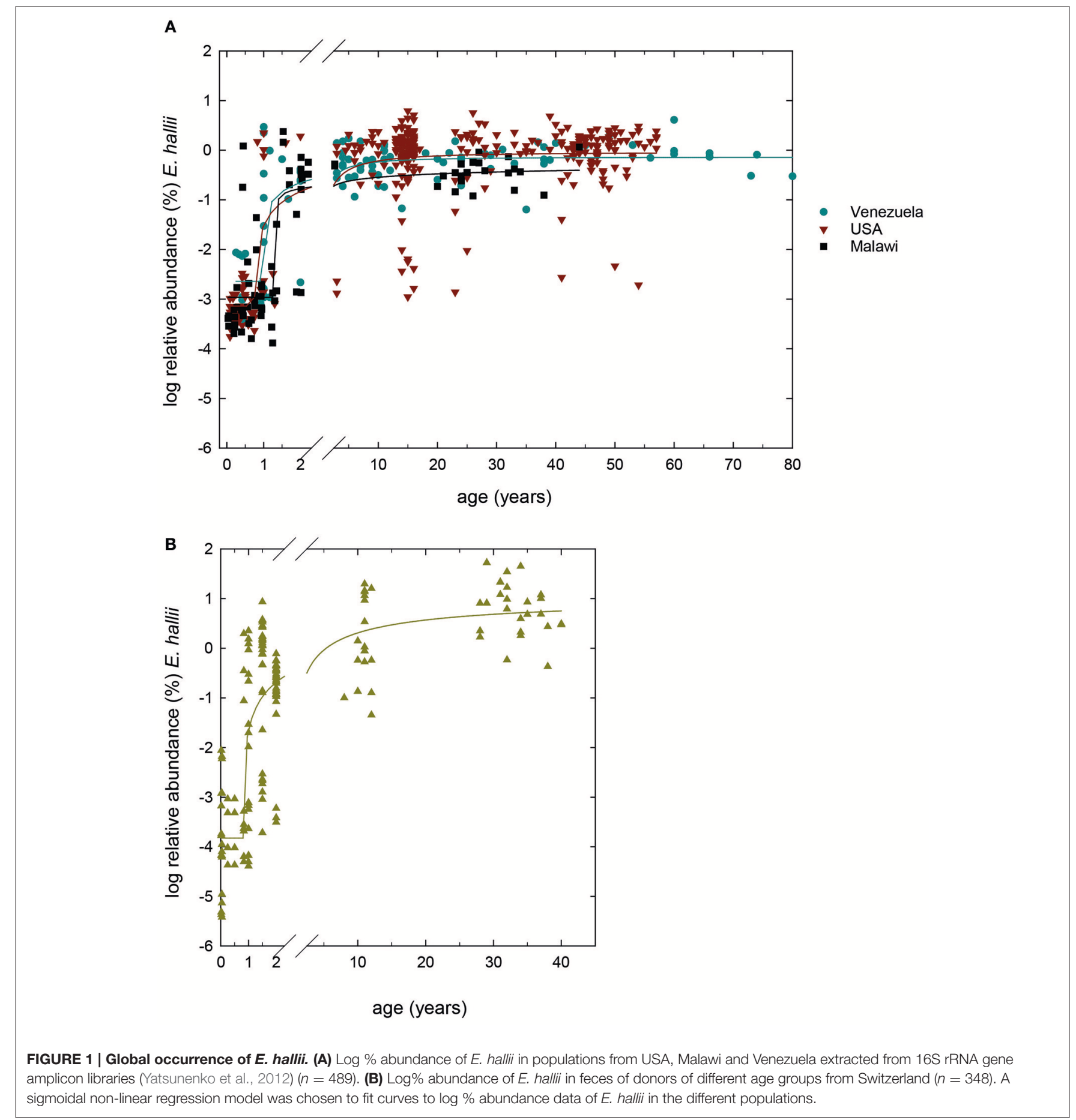


at means of $\log -2.0 \pm 1.2,-2.9 \pm 1.0$, and $-2.9 \pm 0.8 \%$, respectively (Figure 1A). Relative abundance increased after 11.5 years in donors from Venezuela (100\% occurrence), the USA (95\% occurrence), and Malawi (100\%). In Swiss infants, occurrence levels of $E$. hallii fluctuated between 13 and $40 \%$ until 1 year, and increased to $85 \%$ at 2 years of age $(\log -2.9 \pm$ $0.8 \%$; Figure 1B). Between 5 and 10 years, relative abundance of E. hallii reached adult levels in all four populations (Malawi: log $-0.4 \pm 0.3 \%$, Venezuela: $\log -0.2 \pm 0.3 \%$, USA: $\log -0.1 \pm 0.6 \%$, Switzerland: $\log 0.8 \pm 0.5 \%)$.

\section{Growth of $E$. hallii and $B$. longum subsp. Infantis in Single- and Co-Cultures}

To investigate trophic interactions between B. longum subsp. infantis and E. hallii in the presence of glucose, L-fucose and FL, B. longum subsp. infantis, and E. hallii were grown in single- and co-cultures in mYCFA.

In single culture, B. longum subsp. infantis reached a final optical density $\left(\mathrm{OD}_{600 \mathrm{~nm}} 1.9\right)$ in the presence of glucose, and a lower maximal $\mathrm{OD}_{600 \mathrm{~nm}}$ when grown with L-fucose and FL (1.5 \pm 0.1 and 1.5, respectively) after $48 \mathrm{~h}$ of incubation (Figure $2 \mathrm{~A}$ ). In mYCFA_glc - $25.6 \mathrm{mM}$ glucose was used, and 55.4, 9.9, and $13.1 \mathrm{mM}$ acetate, lactate, and formate were formed (Figure 2D). In mYCFA_fuc, B. longum subsp. infantis used $-28.3 \pm 2.8 \mathrm{mM}$ L-fucose and produced nearly equimolar amounts of 1,2-PD, acetate and formate $(25.5 \pm 4.8,22.4 \pm 1.0$, and $26.7 \pm 0.5 \mathrm{mM}$, respectively; Figure 2G). Lactate was produced at low amounts $(2.0 \pm 1.0 \mathrm{mM})$, and mean carbon recovery was $86 \%$. In the presence of FL, B. longum subsp. infantis formed acetate (33.6 $\mathrm{mM})$, lactate $(16.8 \mathrm{mM}), 1,2-\mathrm{PD}(7.8 \mathrm{mM})$, and low amounts of formate (3.1 mM; Figure 2J).

In single culture, E. hallii grew rapidly to $\mathrm{OD} 600 \mathrm{~nm}$ of 1.7 \pm 0.2 after $12 \mathrm{~h}$ of incubation in mYCFA_glc (Figure 2B). From $1 \mathrm{~mol}$ glucose and $0.5 \mathrm{~mol}$ acetate, $\sim 1 \mathrm{~mol}$ butyrate and $0.5 \mathrm{~mol}$ formate were produced (Figure 2E). Maximum optical density in mYCFA_FL and mYCFA_fuc was significantly lower than with glucose with $\mathrm{OD}_{600 \mathrm{~nm}} 0.9$ and 0.5 after 12 and $9 \mathrm{~h}$ of incubation, respectively (Figure 2B). With L-fucose and FL, E. hallii formed $2.7 \pm 1.0$ and $6.0 \pm 1.1 \mathrm{mM}$ butyrate, respectively (Figures $\mathbf{2 H}, \mathbf{K}$ ). No acetate utilization and formate production were detected.

For the co-cultures, qPCR was used in addition to optical density measurements to monitor the growth of both strains (Figure 3). Cell counts were calculated based on 16S rRNA genes corrected for several $16 \mathrm{~S}$ rRNA gene copies per genome. In mYCFA_glc, cell counts of both strains increased by $\log 2.8$ cells $\mathrm{ml}^{-1}$ during the first $8 \mathrm{~h}$ of incubation, after which growth slowed down (Figure 3A). Highest $\mathrm{OD}_{600} \mathrm{~nm}$ was reached after $24 \mathrm{~h}$ of incubation (Figure 2C). The amount of acetate used, and butyrate and formate formed in the co-cultures was similar to $E$. hallii single cultures (Figure 2F), and only low amounts of lactate $(0.6 \pm 0.7 \mathrm{mM})$ were detected after $48 \mathrm{~h}$ (Figure 2F).

Co-cultures grown in $\mathrm{mYCFA}$ _fuc reached a maximum OD of $1.1 \pm 0.1$ after $48 \mathrm{~h}$ of incubation (Figure 2C). During the first $12 \mathrm{~h}, B$. longum subsp. infantis and $E$. hallii grew exponentially with increased cell counts of $\log 3$ and $\log 2.7$ cells $\mathrm{mL}^{-1}$, respectively (Figure 3B). In co-cultures, B. longum subsp. infantis utilized only $\sim 35 \%$ of L-fucose compared to single cultures $(10.4 \pm 8 \mathrm{mM})$. The co-cultures formed propionate $(8.2 \pm 3.5$ $\mathrm{mM})$, butyrate $(7.4 \pm 0.8 \mathrm{mM})$, and formate $(10.9 \pm 1.7 \mathrm{mM}$; Figure 2I). Butyrate formed by the co-cultures was significantly $(p<0.05)$ higher compared to butyrate formation of $E$. hallii single culture in mYCFA_fuc $(7.4 \pm 0.8$ vs. $2.7 \pm 1.0 \mathrm{mM})$. 1,2-PD, propanal, and propanol were not detected during coculture fermentations, and there was no apparent accumulation or consumption of acetate or lactate (Figure 2I).

When grown in co-cultures in mYCFA_FL, cell counts of B. longum subsp. infantis and E. hallii increased by 3.2 and $2.8 \log$ during the first $8 \mathrm{~h}$ of incubation (Figure 3C), and maximal OD was reached after $24 \mathrm{~h}$ of incubation (Figure 2C). Acetate (18.2 $\pm 1.6 \mathrm{mM})$, propionate $(9.7 \pm 5.1 \mathrm{mM})$, and butyrate $(5.4 \pm 3.1$ $\mathrm{mM})$, and formate $(19.7 \pm 5.0 \mathrm{mM})$ were detected after $48 \mathrm{~h}$ of incubation (Figure 2L). 1,2-PD and lactate were only present at low levels $<1 \mathrm{mM}$.

\section{Bifidobacterium Production of 1,2-PD from L-Fucose}

To investigate whether L-fucose utilization was a trait limited to B. longum subsp. infantis, or was also present in other infant-, adult-, or animal-associated bifidobacteria, we screened $n=56$ Bifidobacterium strains (Table 2) for growth in API medium supplied with $30 \mathrm{mM} \mathrm{L}$-fucose and trace amounts of glucose. All isolates were capable of growing in API medium supplied with 30 $\mathrm{mM}$ glucose indicating the suitability of the assay. Only strains of $B$. longum subsp. infantis and subsp. suis, and of B. breve were identified as being able to metabolize L-fucose and to form 1,2PD. L-fucose was used and 1,2-PD was formed in a ratio of $\sim 1: 1$ by all L-fucose-utilizing strains (Table 3). Additionally, lactate, acetate, and formate were produced leading to carbon recoveries between 87 and 109\% (Table 3).

\section{Genes Related to L-Fucose Utilization in B. breve Genomes}

We previously identified two genomic regions that encompass genes potentially involved in L-fucose utilization of strains of B. longum subsp. infantis and B. longum subsp. suis (Bunesova et al., 2016). The genome of B. breve DSM 20213 (PRJDB57) possessed a set of genes with slightly differing genome organization to B. longum subsp. infantis and B. longum subsp. suis (Figure 4). Region 1 encompassed a gene encoding an $\alpha$-fucosidase (GH family 95) with $78 \%$ homology in $607 / 783$ AA to BLON_2335 of B. longum subsp. infantis DSM 20088. Region 1 also contained genes encoding a putative L-fuconate dehydratase (96\% homology in $284 / 293$ AA to B. longum subsp. infantis DSM 20088 L-fuconate dehydratase, region 1), a L-fucose dehydrogenase (93\% homology in 245/262 AA, region 1), L-fuconolactone hydrolase (50\% homology in 132/264 $\mathrm{AA}$, region 2), and a 4-hydroxy-tetrahydrodipicolinate synthase (89\% homology in 265/296 AA, region 1). B. breve region 2 contained another L-fuconate dehydratase ( $97 \%$ homology in 417/426 AA, region 2), L-fucose dehydrogenase ( $94 \%$ homology in $249 / 263 \mathrm{AA}$, region 2), a putative fucose permease $(62 \%$ 


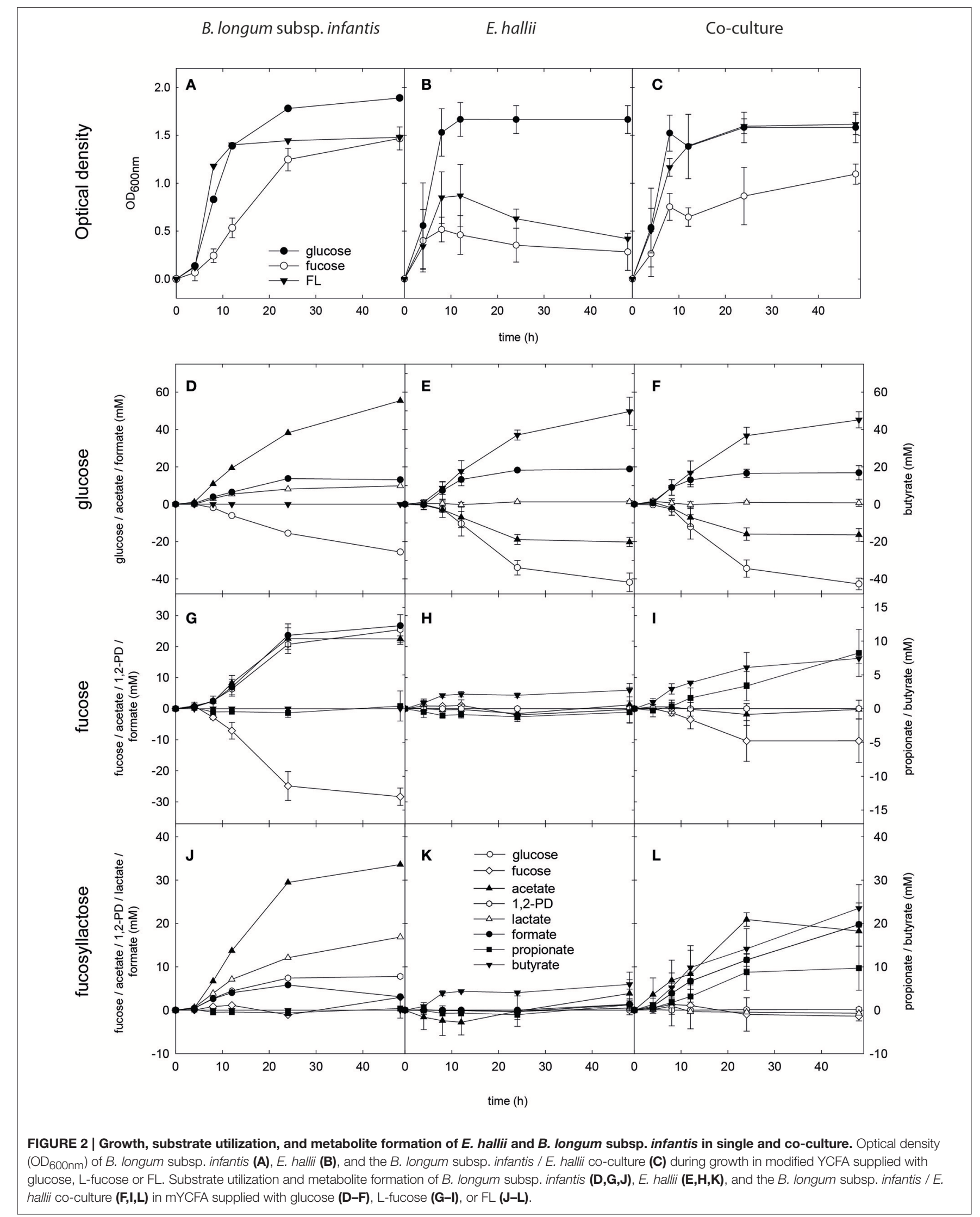




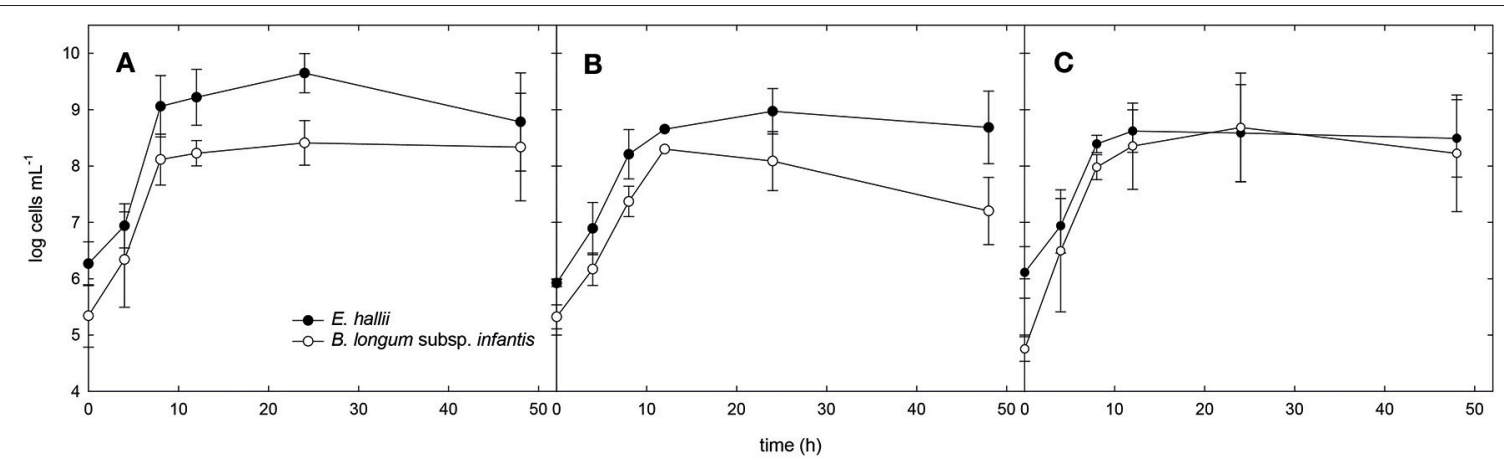

FIGURE 3 | Cell counts of $\boldsymbol{B}$. longum subsp. infantis and $\boldsymbol{E}$. hallii during growth in co-culture. Cell counts of $B$. longum subsp. infantis and $E$. hallii during growth in co-culture in mYCFA_glc (A), mYCFA_fuc (B), and mYCFA_FL (C).

TABLE 3 | Substrate utilization, metabolite formation and carbon recovery.

\begin{tabular}{|c|c|c|c|c|c|c|c|}
\hline \multirow[b]{2}{*}{ Species } & \multirow[b]{2}{*}{ ID } & \multirow{2}{*}{$\begin{array}{l}\text { Substrate consumption (mM) } \\
\text { L-fucose }\end{array}$} & \multicolumn{4}{|c|}{ Metabolite production (mM) } & \multirow[t]{2}{*}{ Carbon recovery (\%) } \\
\hline & & & Lactate & Formate & Acetate & 1,2-PD & \\
\hline \multirow[t]{6}{*}{ B. breve } & TPY 10-1 & $-14.9 \pm 4.5$ & $10.2 \pm 5.2$ & $6.3 \pm 6.0$ & $9.2 \pm 6.7$ & $13.8 \pm 4.0$ & 109 \\
\hline & BSM 1-2 & $-8.5 \pm 1.1$ & $5.3 \pm 4.4$ & $4.2 \pm 3.0$ & $3.1 \pm 2.0$ & $9.3 \pm 1.3$ & 105 \\
\hline & TPY 5-1 & $-12.7 \pm 0.5$ & $6.5 \pm 2.3$ & $5.4 \pm 0.9$ & $6.5 \pm 4.1$ & $11.4 \pm 1.4$ & 94 \\
\hline & N4-BM5-i12 & $-8.3 \pm 3.9$ & $4.6 \pm 2.5$ & $4.0 \pm 0.5$ & $2.5 \pm 3.4$ & $6.8 \pm 1.9$ & 87 \\
\hline & N4-NF3-i1 & $-11.7 \pm 3.1$ & $7.2 \pm 1.5$ & $6.6 \pm 6.7$ & $5.5 \pm 5.0$ & $11.2 \pm 2.8$ & 99 \\
\hline & N4-NF3-i3 & $-5.9 \pm 1.4$ & $6.1 \pm 1.5$ & $0.6 \pm 1.1$ & $1.4 \pm 0.9$ & $5.7 \pm 1.7$ & 102 \\
\hline \multirow[t]{2}{*}{ B. longum subsp. infantis } & DSM 20088 & $-3.9 \pm 0.7$ & $3.8 \pm 0.4$ & $-0.1 \pm 0.2$ & $0.8 \pm 1.4$ & $3.9 \pm 0.8$ & 109 \\
\hline & TPY 12-1 & $-3.8 \pm 1.5$ & $1.4 \pm 0.5$ & $1.2 \pm 1.1$ & $1.6 \pm 0.3$ & $3.8 \pm 1.1$ & 91 \\
\hline B. longum subsp. Suis & BSM 11-5 & $-11.5 \pm 1.0$ & $5.8 \pm 1.4$ & $4.8 \pm 4.2$ & $3.3 \pm 0.9$ & $11.2 \pm 1.3$ & 91 \\
\hline
\end{tabular}

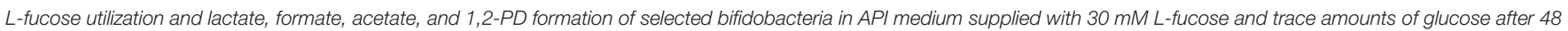
$h$ of incubation at $37^{\circ} \mathrm{C}$.

homology in 262/461 AA, region 2), a L-fuconolactone hydrolase (96\% homology in 246/255 AA, region 2), and a 4-hydroxytetrahydrodipicolinate synthase (69\% homology in 206/296 AA, region 1; Figure 4). B. breve UCC 2003 had an identical genomic set-up to B. breve DSM 20213 (data not shown).

\section{Coding Potential for Bifidobacteria Fucose Utilization in Fecal Metagenomes}

Bifidobacteria were the predominant taxa in feces of infants from Venezuela, Malawi, and the USA during the first 2 years of life based on 16S rRNA gene sequencing data (Figure 5). For 111 samples, metagenomic data of the same donor data were also available (Yatsunenko et al., 2012). Metagenomes were screened for the presence of proteins presumably involved in bifidobacterial L-fucose utilization (Figure 4). At the threshold of detection allowed by sequencing coverage, the majority of fecal metagenomes of infants under 2 years of all three populations possessed the coding potential for bifidobacterial fucose utilization (Figure 5). All infant metagenomes from Venezuela ( $n=11,0.25-2$ years) had coding potential for bifidobacteria L-fucose utilization. Eighty-seven and Seventy-one percent of proteins of both regions were recovered for B. longum subsp. infantis and $B$. breve respectively; all infants were positive for $B$. longum subsp. infantis while $B$. breve L-fucose utilization was not detected in 1 infant. Similarly, all infant metagenomes from Malawi ( $n=18,0.05-1.53$ years) had coding potential for bifidobacteria L-fucose utilization. On average, 93 and $74 \%$ of the proteins of B. longum subsp. infantis and B. breve were detected, respectively. Of the 44 fecal metagenomes of US American infants (0.08-1.6 years), 16 and 28 were negative and positive for bifidobacteria L-fucose utilization, respectively. B. breve assigned proteins were detected in all children while B. longum subsp. infantis L-fucose utilization related proteins were not detected in one infant. The recovery of proteins of region 1 and 2 was 47 and $23 \%$ for B. longum subsp. infantis and B. breve, respectively. Bifidobacterial L-fucose utilization related proteins were also detected in feces of a 5 and a 6 year old US American child (Figure 5).

\section{DISCUSSION}

E. hallii is a metabolically versatile species that can contribute to intestinal butyrate and propionate formation (Duncan et al., 2004; Engels et al., 2016). In adults, E. hallii is a regular constituent of the gut microbiota (Engels et al., 2016). As shown in this study, E. hallii persistently occurred in the first months 


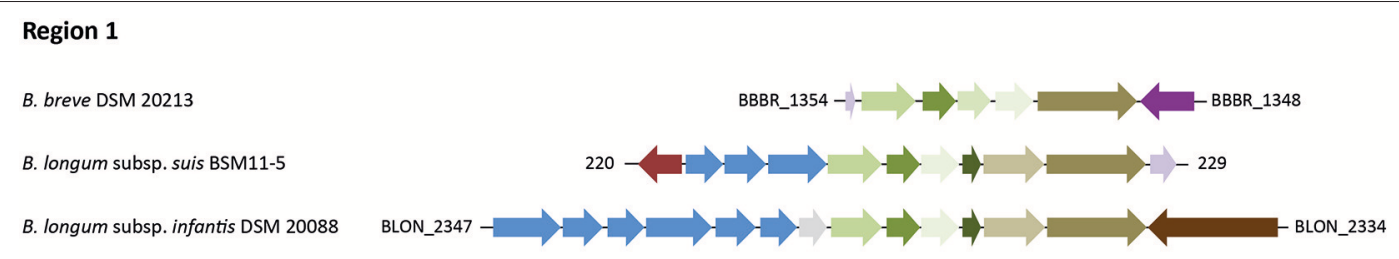

Region 2

B. breve DSM 20213

B. longum subsp. suis BSM11-5

B. longum subsp. infantis DSM 20088
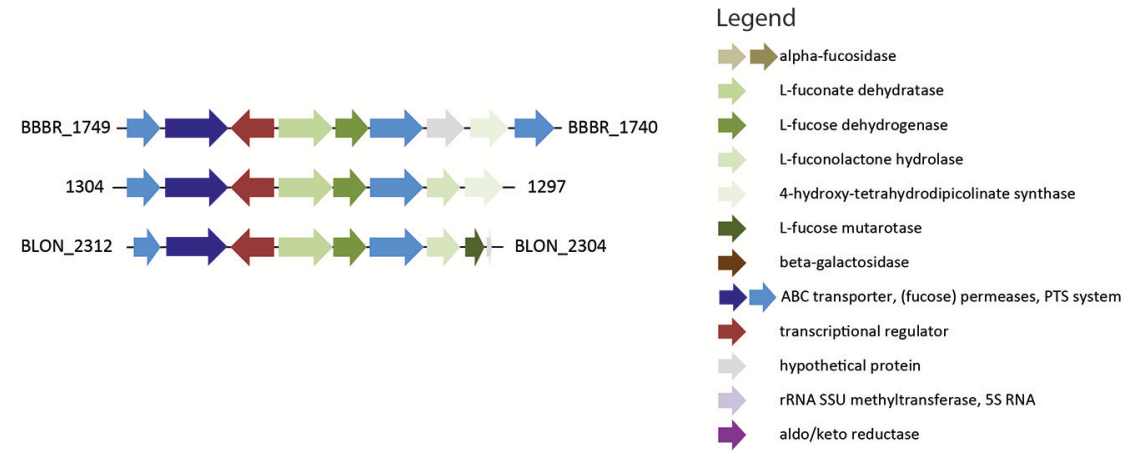

FIGURE 4 | Genomic regions encompassing genes putatively involved in L-fucose degradation in $\boldsymbol{B}$. longum and $\boldsymbol{B}$. breve. The gene cluster of region 1 also contained genes encoding the $\alpha$-fucosidases BLON_2335 and BLON_2336 and is part of the B. longum subsp. infantis HMO utilization operon H1 (adapted from LoCascio et al., 2010; Bunesova et al., 2016 not drawn according to scale).

after birth at low abundance of the fecal microbiota and reached adult levels at $\sim 5-10$ years of age independent of geographical donor origin. Thus, E. hallii is a commensal occurring very early in life which might contribute to metabolic interactions starting at 1-2 years of age when abundance markedly increased in all populations.

$B$. breve and $B$. infantis subsp. longum were identified as species capable of metabolizing fucose. Both are two of the most representative species found in breast-milk fed infants (Avershina et al., 2013; Matsuki et al., 2016), and fecal metagenome analysis indicated the coding potential for bifidobacterial fucose utilization in children under 2 years. Trophic interactions of $E$. hallii and B. longum subsp. infantis and/or B. breve during L-fucose and FL utilization can therefore be considered infant specific (Figure 6). Interestingly, general occurrence and the presence of bifidobacteria capable of L-fucose utilization appeared to be higher in Venezuelan and Malawian than in American infants. This might reflect differences in feeding practice, as in Venezuela and Malawi, all analyzed infants below 2 years were breast-fed, while in the USA, 71\% received formula (Yatsunenko et al., 2012).

In the infant gut, E. hallii can utilize lactate and acetate produced by bifidobacteria during the degradation of hexoses. Bifidobacteria metabolize hexoses via the "bifid shunt" with fructose-6-phosphoketolase as the key enzyme. Glucose (1 mol) theoretically yields $1.5 \mathrm{~mol}$ acetate, $1 \mathrm{~mol}$ lactate, and 2.5 ATP (de Vries and Stouthamer, 1967, 1968). However, this ratio depends on whether the intermediate pyruvate is cleaved to acetyl phosphate and formate, or whether it is reduced to lactate (Palframan et al., 2003). B. longum subsp. infantis, B. longum subsp. suis, and $B$. breve also metabolized the desoxyhexose
L-fucose. In mYCFA, nearly equimolar proportions of acetate, formate and 1,2-PD and only little lactate were formed while in API medium, lactate was produced at the expense of formate and acetate. The ratio of acetate, formate, and lactate formed varies for species, substrate source and carbohydrate supply (limitation or excess; Macfarlane and Gibson, 1995; Palframan et al., 2003). Here, ratios differed for the same species when supplied with the same carbohydrate (30 mM L-fucose). In this study, mYCFA was prepared strict anaerobically while API medium was only facultative anaerobic, which could have also impacted pyruvate metabolism. It was shown before that the presence of oxygen changed final metabolites formed by B. longum subsp. infantis (González et al., 2004).

Both bifidobacteria and $E$. hallii were able to produce formate during the degradation of FL (Figure 6). Methanogens, which can produce methane from formate and $\mathrm{CO}_{2}$, are usually not detected in infants (Vanderhaeghen et al., 2015). As feces collected as part of a cohort study following 16 infants from 2 weeks to 2 years of age (Pham et al., 2016, unpublished data) contained no or only very low levels of formate, it can be assumed that the formate produced during $\mathrm{HMO}$ degradation is further utilized. Formate together with $\mathrm{CO}_{2}$ can also be used by acetogenic microbes such as Blautia spp. to produce acetate via the Wood-Ljungdal pathway. Little literature exists on formate cross-feeding within the infant gut microbiota. However, as one of the important intermediate metabolites, the effect of formate and formate utilization on infant gut health should be investigated in further studies.

We identified for the first time the ability of $B$. breve strains to metabolize L-fucose to 1,2-PD. We previously 


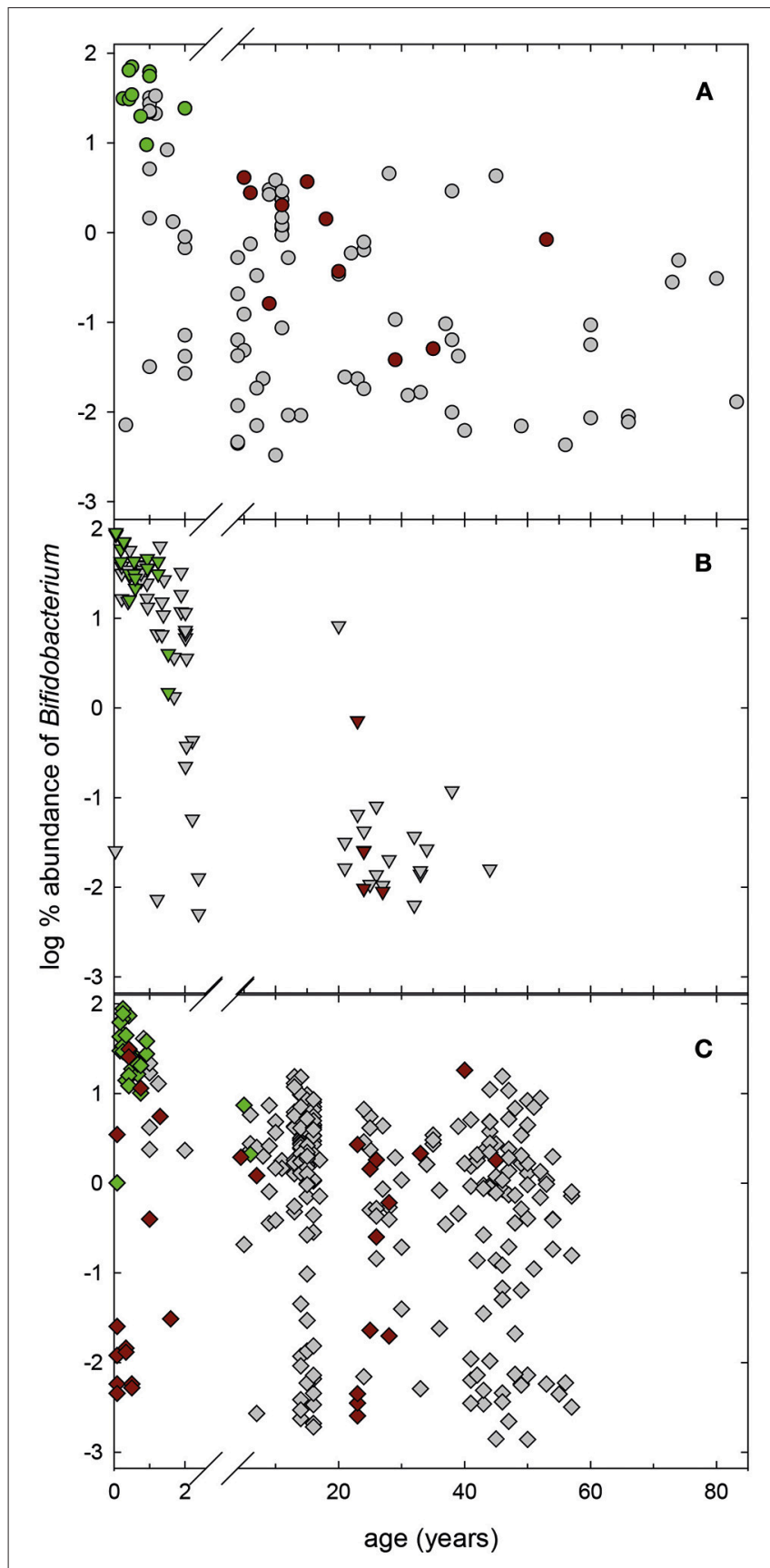

FIGURE 5 | Relative abundance of the genus Bifidobacterium in infant fecal samples. Relative abundance of Bifidobacterium in 16S rRNA gene libraries of infant feces collected from Venezuela (A, $n=98)$, Malawi (B, $n=$ 84), and USA (C, $n=307$; Yatsunenko et al., 2012). For samples with available metagenomes (Yatsunenko et al., 2012), the presence of genes encoding bifidobacterial L-fucose utilization related proteins. Green symbols, samples that were positive for bifidobacteria L-fucose utilization related proteins, red symbols, samples that were negative, gray samples, no metagenomes available.

suggested a Bifidobacterium L-fucose utilization pathway based on genome comparison which has been also identified by a recent study investigating FL degradation by a strain of B. longum subsp. longum (Bunesova et al., 2016; Garrido et al., 2016). Similar to strains of B. longum subsp. infantis and B. longum subsp. suis, $B$. breve harbored two genomic regions which encompassed genes putatively encoding enzymes involved in L-fucose degradation with non-phosphorylated intermediates. In contrast, the L-fucose-negative $B$. kashiwanohense only possessed region 1 (Bunesova et al., 2016), suggesting that both regions are necessary for L-fucose metabolism. In addition, two of the identified genes encoding fucose permease (FucP) and a 4-hydroxy-tetrahydrodipicolinate synthase, were upregulated when $B$. breve was grown in co-culture with mucin degrading $B$. bifidum (Egan et al., 2014). B. bifidum releases L-fucose during growth with mucin similar to the release of fucose from FL (Turroni et al., 2014; Garrido et al., 2015; Bunesova et al., 2016), which then can be imported and metabolized by other species such as $B$. breve or L-fucose-utilizing strains of B. longum.

In adults, $B$. adolescentis is a predominant Bifidobacterium species, and cross-feeding between B. adolescentis and E. hallii has been reported before (Belenguer et al., 2006). E. hallii was thereby able to form butyrate from lactate and acetate that was produced when $B$. adolescentis grew in the presence of starch, or to use mono- or dissacharides released by B. adolescentis from fructooligosaccharides. We also observed substrate-dependent routes of metabolic cross-feeding. In the presence of L-fucose, propionate was the main metabolite formed by E. hallii. In co-cultures with $B$. longum subsp. infantis, the formation of propionate, butyrate, and formate from FL was observed. Here, butyrate and formate could have been produced from lactate and acetate, or directly from glucose released by B. longum subsp. infantis, while propionate again was derived from L-fucose.

FL is a major component of HMOs (de Leoz et al., 2012). Therefore, it can be implied that a substantial proportion of L-fucose is metabolized to propionate if $E$. hallii is present. However, not all women are able to secrete $\alpha$-(1-2)fucosylated HMOs due to mutations that render the responsible fucosyltransferase FUT2 inactive. About 20\% of the European and African population carry an inactive FUT2 (Kelly et al., 1995; Liu et al., 1998). The activity of FUT2 and with that the presence of fucosylated HMOs has been linked to differences in the establishment of the infant gut microbiota (Lewis et al., 2015). The bifidobacteria community of infants born to nonsecretor mothers was reported to establish later, to carry reduced numbers of bifidobacteria, and to contain a lower percentage of bifidobacteria capable of degrading 2-FL compared to infants of secretor mothers (Lewis et al., 2015). Thus, non-secretor mothers' milk lacks a significant source for intestinal propionate formation. It could therefore be speculated that the SCFA profile of infants of secretor- and non-secretor mothers is different. However, to date, no large scale study compared the fecal SCFA profile of infants born to secretor and non-secretor mothers.

Fucosylated oligosaccharides are not only a carbon source for the intestinal microbes but also play an important role in host-microbe interactions (Pickard et al., 2014). On one hand, fucosylated HMOs protect against invasion of enteric pathogens acting as a decoy for epithelial attachment sites (Morrow et al., 2005). On the other hand, L-fucose released from fucosylated oligosaccharides has been related to virulence and 


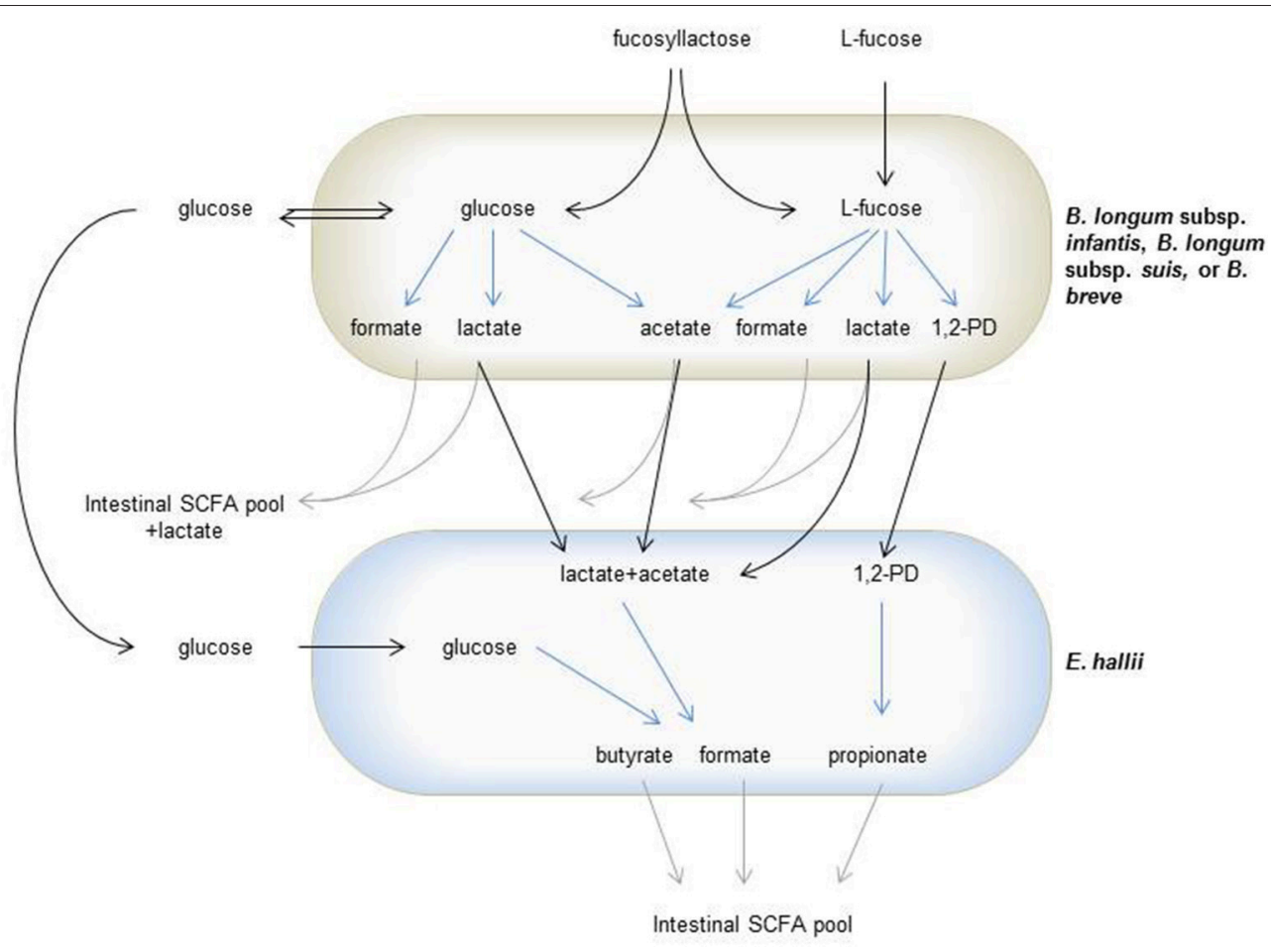

FIGURE 6 | Trophic interactions of L-fucose utilizing bifidobacteria and E. hallii during degradation of glucose, L-fucose or FL. Gray arrows indicate glucose, L-fucose or FL derived degradation metabolites fed in the intestinal SCFA pool, black arrows indicate sugars or metabolites cross-fed between bifidobacteria and $E$. hallii, blue arrows indicate metabolite formation.

host colonization of enteric pathogens. It was suggested that Lfucose released by Bacteroides thetaiotamicron was utilized by Salmonella enterica serovar Typhimurium during colonization of a mouse model ( $\mathrm{Ng}$ et al., 2013). Likewise, L-fucose released by $B$. thetaiotamicron increased colonization of an enterohaemorrhagic E. coli (EHEC) in comparison to mutants with a knock-out of a fucose sensing two-component system (Pacheco et al., 2012). This fucose sensing two-component system regulates virulence and metabolic gene expression of EHEC (Pacheco et al., 2012). The ability to utilize L-fucose provided Campylobacter jejuni with a competitive advantage during the colonization of birds or pigs compared to fucosenegative mutants (Muraoka and Zhang, 2011; Stahl et al., 2011). Through utilization of L-fucose, the infant bifidobacterial community therefore could enhance colonization resistance toward enteric pathogens.

\section{CONCLUSION}

L-fucose utilization was identified here as a trait of only infant-derived bifidobacteria. Trophic interactions of L-fucose-utilizing infant bifidobacteria and E. hallii yielded different SCFAs from L-fucose or FL pointing at E. hallii as a metabolically versatile species utilizing intermediate metabolites of HMO fermentation. The utilization of L-fucose by the infant bifidobacterial community might enhance colonization resistance toward fucose-dependent enteric pathogens.

\section{AUTHOR CONTRIBUTIONS}

VB and CS designed experiments; VB, CS, VTP, and HR conducted experimental work; VB, CS, VTP, and H-JR analyzed data; CL, NB provided financial support; CS wrote manuscript with the help of all authors.

\section{FUNDING}

VB was supported by SCIEX grant 13.151. VTP and this research were supported by a grant of the Swiss National Science Fond (PN 310030_146784). H-JR was funded by the European Research Council (ERC Synergy Grant No. 609883 to NB).

\section{ACKNOWLEDGMENTS}

We thank Glycom A/S for supplying 2'-FL and 3'-FL. Alfonso Die and Nicole Oberortner are acknowledged for technical assistance. 


\section{REFERENCES}

Avershina, E., Storrø, O., Øien, T., Johnsen, R., Wilson, R., Egeland, T., et al. (2013). Bifidobacterial succession and correlation networks in a large unselected cohort of mothers and their children. Appl. Environ. Microbiol. 79, 494-407. doi: 10.1128/aem.02359-12

Becker, D. J., and Lowe, J. B. (2003). Fucose: biosynthesis and biological function in mammals. Glycobiology 13, 41-53. doi: 10.1093/glycob/cwg054

Belenguer, A., Duncan, S. H., Calder, A. G., Holtrop, G., Louis, P., Lobley, G. E., et al. (2006). Two routes of metabolic cross-feeding between Bifidobacterium adolescentis and butyrate-producing anaerobes from the human gut. Appl. Environ. Microbiol. 72, 3593-3599. doi: 10.1128/AEM.72.5.3593-3599.2006

Buchfink, B., Xie, C., and Huson, D. H. (2015). Fast and sensitive protein alignment using DIAMOND. Nat. Methods 12, 59-60. doi: 10.1038/nmeth.3176

Bunesova, V., Lacroix, C., and Schwab, C. (2016). Fucosyllactose and Lfucose utilization of infant Bifidobacterium longum and Bifidobacterium kashiwanohense. BMC Microbiol. 16:248. doi: 10.1186/s12866-016-0867-4

de Leoz, M. L., Gaerlan, S. C., Strum, J. S., Dimapasoc, L. M., Mirmiran, M., Tancredi, D. J., et al. (2012). Lacto-N-tetraose, fucosylation, and secretor status are highly variable in human milk oligosaccharides from women delivering preterm. J. Proteome Res. 11, 4662-4672. doi: 10.1021/pr3004979

de Vries, W., and Stouthamer, A. H. (1967). Pathway of glucose fermentation in relation to the taxonomy of bifidobacteria. J. Bacteriol. 93, 574-576.

de Vries, W., and Stouthamer, A. H. (1968). Fermentation of glucose, lactose, galactose, mannitol, and xylose by bifidobacteria. J. Bacteriol. 96, 472-478.

Duncan, S. H., Louis, P., and Flint, H. J. (2004). Lactate-utilizing bacteria, isolated from human feces, that produce butyrate as a major fermentation product. Appl. Environ. Microbiol. 70, 5810-5817. doi: 10.1128/AEM.70.10.5810-5817.2004

Egan, M., O'Connell Motherway, M., Kilcoyne, M., Kane, M., Joshi, L., Ventura, M., et al. (2014). Cross-feeding by Bifidobacterium breve UCC2003 during cocultivation with Bifidobacterium bifidum PRL2010 in a mucin-based medium. BMC Microbiol. 14:282. doi: 10.1186/s12866-014-0282-7

Engels, C., Ruscheweyh, H.-J., Beerenwinkel, N., Lacroix, C., and Schwab, C. (2016). The common gut microbe Eubacterium hallii also contributes to intestinal propionate formation. Front. Microbiol. 7:713. doi: 10.3389/fmicb.2016.00713

Fierer, N., Jackson, J. A., Vilgalys, R., and Jackson, R. B. (2005). Assessment of soil microbial community structure by use of taxon-specific quantitative PCR assays. Appl. Environ. Microbiol. 71, 4117-4120. doi: 10.1128/AEM.71.7.4117-4120.2005

Garrido, D., Ruiz-Moyano, S., Kirmiz, N., Davis, J. C. C., Totten, S., Lemay, D. G., et al. (2016). A novel gene cluster allows preferential utilization of fucosylated milk oligosaccharides in Bifidobacterium longum subsp. longum SC596. Sci. Rep. 6:35045. doi: 10.1038/srep35045

Garrido, D., Ruiz-Moyano, S., Lemay, D. G., Sela, D. A., German, J. B., and Mills, D. A. (2015). Comparative transcriptomics reveals key differences in the response to milk oligosaccharides of infant gut-associated bifidobacteria. Sci. Rep. 5:13517. doi: 10.1038/srep13517

González, R., Blancas, A., Santillana, R., Azaola, A., and Wacher, C. (2004). Growth and final product formation by Bifidobacterium infantis in aerated fermentations. Appl. Microbiol. Biotechnol. 65, 606-610. doi: 10.1007/s00253-004-1603-9

Herbig, A., Maixner, F., Bos, K. I., Zink, A., Krause, J., and Huson, D. H. (2016). MALT: fast alignment and analysis of metagenomic DNA sequence data applied to the Tyrolean Iceman. bioRxiv 050559. doi: 10.1101/050559

Jost, T., Lacroix, C., Braegger, C. P., Rochat, F., and Chassard, C. (2014). Vertical mother-neonate transfer of maternal gut bacteria via breastfeeding. Environ. Microbiol. 16, 2891-2904. doi: 10.1111/1462-2920.12238

Kelly, R. J., Rouquier, S., Giorgi, D., Lennon, G. G., and Lowe, J. B. (1995). Sequence and expression of a candidate for the human secretor blood group $\alpha(1,2)$ fucosyltransferase gene (FUT2). J. Biol. Chem. 270, 4640-4649. doi: $10.1074 / \mathrm{jbc} .270 .9 .4640$

Kunz, C., Rudloff, S., Baier, W., Klein, N., and Strobel, S. (2000). Oligosaccharides in human milk. Structural, functional, and metabolic aspects. Annu. Rev. Nutr. 20, 699-722. doi: 10.1146/annurev.nutr.20.1.699

Lewis, Z. T., Totten, S. M., Smilowitz, J. T., Popovic, M., Parker, E., Lemay, D. G., et al. (2015). Maternal fucosyltransferase 2 status affects the gut bifidobacterial communities of breasfed infants. Microbiome 3, 13 . doi: 10.1186/s40168-015-0071-z

Liu, Y., Koda, Y., Soejima, M., Pang, H., Schlaphoff, T., du Toit, E. D., et al. (1998). Extensive polymorphism of the FUT2 gene in an African (Xhosa) population of South Africa. Hum. Genet. 103, 204-210. doi: 10.1007/s004390050808

LoCascio, R. G., Desai, P., Sela, D. A., Weimer, B., and Mills, D. A. (2010). Broad conservation of milk utilization genes in Bifidobacterium longum subsp. infantis as revealed by comparative genomic hybridization. Appl. Environ. Microbiol. 76, 7373-7381. doi: 10.1128/AEM.00675-10

Macfarlane, G. T., and Gibson, G. R. (1995). "Microbiological aspects of short chain fatty acid production in the large bowel," in Physiological and Clinical Aspects of Short Chain Fatty Acid Metabolism, eds J. H. Cummings, J. L. Rombeau, and T. Sakata (Cambridge: Cambridge University Press), 87-105.

Matsuki, T., Yahagi, K., Mori, H., Matsumoto, H., Hara, T., Tajima, S., et al. (2016). A key genetic factor for fucosyllactose utilization affects infant gut microbiota development. Nat. Commun. 7:11939. doi: 10.1038/ncomms11939

Morrow, A. L., Ruiz-Palacios, G. M., Jiang, X., and Newburg, D. S. (2005). Human-milk glycans that inhibit pathogen binding protect breastfeeding infants against infectious diarrhea. J. Nutr. 135, 1304-1307. doi: 10.1146/annurev.nutr.25.050304.092553

Muraoka, W. T., and Zhang, Q. (2011). Phenotypic and genotypic evidence for L-fucose utilization by Campylobacter jejuni. J. Bacteriol. 193, 1065-1075. doi: 10.1128/JB.01252-10

Ng, K. M., Ferreyra, J. A., Higginbottom, S. K., Lynch, J. B., Kashyap, P. C., Gopinath, S., et al. (2013). Microbiota-liberated host sugars facilitate post-antibiotic expansion of enteric pathogens. Nature 502, 96-99. doi: $10.1038 /$ nature 12503

Niñonuevo, M. R., Perkins, P. D., Francis, J., Lamotte, L. M., LoCascio, R. G., Freeman, S. L, et al. (2008). Daily variations in oligosaccharides of human milk determined by microfluidic chips and mass spectrometry. J. Agric. Food Chem. 56, 618-626. doi: 10.1021/jf071972u

Pacheco, A. R., Curtis, M. M., Ritchie, J. M., Munera, D., Waldor, M. K., Moreira, C. G., et al. (2012). Fucose sensing regulates bacterial intestinal colonization. Nature 492, 113-117. doi: 10.1038/nature11623

Palframan, R. J., Gibson, G. R., and Rastall, R. A. (2003). Carbohydrate preferences of Bifidobacterium species isolated from the human gut. Curr. Issues Intest. Microbiol. 4, 71-75.

Pham, V. T., Lacroix, C., Braegger, C. P., and Chassard, C. (2016). Early colonization of functional groups of microbes in the infant gut. Environ. Microbiol. 18, 2246-2258. doi: 10.1111/1462-2920.13316

Pickard, J. M., Maurice, C. F., Kinnebrew, M. A., Abt, M. C., Schenten, D., Golovkina, T. V., et al. (2014). Rapid fucosylation of intestinal epithelium sustains host-commensal symbiosis in sickness. Nature 514, 638-641 doi: 10.1038/nature13823

Plöger, S., Stumpff, F., Penner, G. B., Schulzke, J. D., Gabel, G., Martens, H., et al. (2012). Microbial butyrate and its role for barrier function in the gastrointestinal tract. Ann. N.Y. Acad. Sci. 1258, 52-59. doi: 10.1111/j.1749-6632.2012.06553.x

Pruitt, K. D., Tatusova, T., and Maglott, D. R. (2007). NCBI reference sequences (RefSeq): a curated non-redundant sequence database of genomes, transcripts and proteins. Nucleic Acids Res. 35, D61-D65. doi: 10.1093/nar/gkl842

Quast, C., Pruesse, E., Yilmaz, P., Gerken, J., Schweer, T., Yarza, P., et al. (2013). The SILVA ribosomal RNA gene database project: improved data processing and web-based tools. Nucleic Acids Res. 41, D590-D596. doi: 10.1093/nar/ gks1219

Ramirez-Farias, C., Slezak, K., Fuller, Z., Duncan, A., Holtrop, G., and Louis, P. (2009). Effect of inulin on the human gut microbiota: stimulation of Bifidobacterium adolescentis and Faecalibacterium prausnitzii. Br. J. Nutr. 101, 541-550. doi: 10.1017/S0007114508019880

Reichardt, N., Duncan, S. H., Young, P., Belenguer, A., McWilliam Leitch, C., Scott, K. P., et al. (2014). Phylogenetic distribution of three pathways for propionate production within the human gut microbiota. ISME J. 8, 1323-1335. doi: 10.1038/ismej.2014.14

Rinttilä, T., Kassinen, A., Malinen, E., Krogius, L., and Palva, A. (2004). Development of an extensive set of $16 \mathrm{~S}$ rDNA-targeted primers for quantification of pathogenic and indigenous bacteria in faecal samples by real-time PCR. J. Appl. Microbiol. 97, 1166-1177. doi: 10.1111/j.1365-2672.2004.02409.x 
Rockova, S., Rada, V., Nevoral, J., Marsik, P., Vlkova, E., and Bunesova, V. (2012). Inter-species differences in the growth of bifidobacteria cultured on human milk oligosaccharides. Folia Microbiol. 57, 321-324. doi: 10.1007/s12223-012-0134-5

Sela, D. A., Garrido, D., Lerno, L., Wu, S., Eom, H.J., Joachimiak, A., et al. (2012). Bifidobacterium longum subsp. infantis ATCC $15697 \alpha$-fucosidases are active on fucosylated human milk oligosaccharides. Appl. Environ. Microbiol. 78, 795-803. doi: 10.1128/AEM.06762-11

Stahl, M., Friis, L. M., Nothaft, H., Liu, X., Li, J., and Szymanski, C. M. (2011). Lfucose utilization provides Campylobacter jejuni with a competitive advantage. Proc. Natl. Acad. Sci. U.S.A. 108, 7194-7199. doi: 10.1073/pnas.10141 25108

Staib, L., and Fuchs, T. M. (2014). From food to cell: nutrient exploitation strategies of enteropathogens. Microbiol. 160, 1020-1039. doi: 10.1099/mic.0.078105-0

Turroni, F., Duranti, S., Bottacini, F., Guglielmetti, S., Van Sinderen, D., and Ventura, M. (2014). Bifidobacterium bifidum as an example of a specialized human gut commensal. Front. Microbiol. 5:437. doi: 10.3389/fmicb.2014.00437

Vanderhaeghen, S., Lacroix, C., and Schwab, C. (2015). Methanogen communities in stools of humans of different age and health status and co-occurrence with bacteria. FEMS Microbiol. Lett. 362:fnv092. doi: 10.1093/femsle/ fnv092
Větrovský, T., and Baldrian, P. (2013). The variability of the 16S rRNA gene in bacterial genomes and its consequences for bacterial community analyses. PLoS ONE 8:e57923. doi: 10.1371/journal.pone.0057923

Wong, J. M., deSouza, R., Kendall, C. W., Emam, A., and Jenkins, D. J. (2006). Colonic health: fermentation and short chain fatty acids. J. Clin. Gastroenterol. 40, 235-243. doi: 10.1097/00004836-200603000-00015

Yatsunenko, T., Rey, F. E., Manary, M. J., Trehan, I., Dominguez-Bello, M. G., Contreras, M., et al. (2012). Human gut microbiome viewed across age and geography. Nature 486, 222-228. doi: 10.1038/nature11053

Conflict of Interest Statement: The authors declare that the research was conducted in the absence of any commercial or financial relationships that could be construed as a potential conflict of interest.

Copyright (C) 2017 Schwab, Ruscheweyh, Bunesova, Pham, Beerenwinkel and Lacroix. This is an open-access article distributed under the terms of the Creative Commons Attribution License (CC BY). The use, distribution or reproduction in other forums is permitted, provided the original author(s) or licensor are credited and that the original publication in this journal is cited, in accordance with accepted academic practice. No use, distribution or reproduction is permitted which does not comply with these terms. 